\title{
Alterações ortopédicas em crianças e adolescentes obesos
}

\author{
Orthopedic alterations in obese children and adolescents
}

\author{
Michelle Brandalize ${ }^{[a]}$, Neiva Leite ${ }^{[b]}$ \\ [a] Mestre, professora do departamento de Fisioterapia da Faculdade Guairacá, Guarapuava, PR - Brasil, e-mail: michelleb_fisio@ \\ yahoo.com.br \\ [b] Doutora em Saúde da Criança e Adolescente pela Universidade Federal do Paraná (UFPR) e líder do Núcleo de Pesquisa em \\ Qualidade de Vida, Curitiba, PR - Brasil, e-mail: neivaleite@gmail.com
}

\section{Resumo}

Introdução: A obesidade infanto-juvenil é um problema muito frequente e implica complicações que podem estar presentes precocemente, como as alterações ortopédicas. Objetivo: descrever as alterações que atingem o aparelho locomotor em crianças e adolescentes com sobrepeso e obesidade. Metodologia: Foram consultadas as bases de dados MEDLINE, SciELO, LILACS, ISI e base de dados da Universidade de Standford, com a combinação dos seguintes descritores: "obesidade", "complicações", "criança", "dores músculo-esqueléticas", "postura", "alterações ortopédicas", "marcha" e seus respectivos sinônimos em inglês. Resultados: Pôde-se observar que as crianças e adolescentes obesos são mais predispostos a apresentar essas complicações ortopédicas que os indivíduos eutróficos, e os principais problemas relatados foram as alterações posturais, como hiperlordose lombar e joelhos valgos, e dores músculo-esqueléticas, principalmente na coluna lombar e membros inferiores. Os principais fatores que causam esses problemas não são bem definidos, mas incluem aumento da sobrecarga articular associado à fragilidade óssea em fase de crescimento e à diminuição da estabilidade postural, o que conduz ao aumento das necessidades mecânicas regionais. Conclusão: A identificação precoce dessas disfunções é fundamental para que essas crianças recebam tratamento multidisciplinar adequado.

Palavras-chave: Obesidade na adolescência. Obesidade infantil. Alterações ortopédicas. Dor músculo-esquelética.

\begin{abstract}
Introducion: Childhood obesity is a very recurrent problem that causes some complications which can be present in early stage oflife, like orthopedic alterations. Objective: To describe the locomotor system alterations in obese children
\end{abstract}


and adolescents. Methods: Articles were found in MEDLINE, SciELO, LILACS, ISI and Standford databases. The follow key-words were used: "children obesity", "orthopedic disorders", "posture", "gait" and "musculoskeletal pain", in Portuguese and English. Results: This review could observe that obese children and adolescents are more predispose to have these complications than the eutrofic ones and the main problems are the postural alterations, like lumbar hiperlordosis and genu valgum. Musculoskeletal pains are also a common complaint, mainly back pain and knee pain. The main factors that can cause these problems are not well defined, but include an increase in body fat mass and in weight-bearing forces associated to a weaker bone in growth phase and the decrease in postural stability, which leads to a higher mechanical regional needs are some hypothesis. Conclusion: The early identification of these problems is essential for these children to receive the adequate multi-professional care.

Keywords: Adolescent obesity. Childhood obesity. Orthopedic disorders. Musculoskeletal pain.

\section{Introdução}

O excesso de peso na infância e na adolescência aumentou em nosso país em função das modificações nos hábitos de vida, como o sedentarismo e o consumo de alimentos inadequados (1). A obesidade é uma doença crônica e inter-relacionada direta ou indiretamente com outras situações patológicas (2), e entre as suas complicações destacam-se aquelas relacionadas à síndrome metabólica $(3,4)$. No entanto, outras alterações, como problemas respiratórios, dermatológicos e ortopédicos, também podem ocorrer e são pouco pesquisados (5).

A prevalência brasileira de adolescentes com excesso de peso é de $16,7 \%$, sendo mais comum em meninos que em meninas, e a região Sul apresenta o maior índice do país (6). As alterações no sistema cardiovascular foram pesquisadas em jovens de Curitiba e descobriu-se que os obesos apresentam índices mais elevados de pressão arterial, dislipidemias e resistência à insulina, quando comparados com os não obesos (7).

Diferente do que acontece com os estudos na área de complicações metabólicas na criança obesa, os estudos relacionados aos problemas ortopédicos são mais escassos, porém são encontrados relatos de alterações posturais, dores músculo-esqueléticas e doenças como a epifisiólise da cabeça femoral, as osteocondrites e a tíbia vara como os mais frequentes (8).

Em adultos, a obesidade está relacionada às doenças articulares degenerativas (9). Acredita-se que um aumento na sobrecarga causado pela obesidade pode afetar as articulações do membro inferior, causando desalinhamento e dor em idades precoces (10).

Dessa forma, o objetivo deste estudo é descrever as alterações que atingem o aparelho locomotor em crianças e adolescentes com sobrepeso e obesidade, baseando-se em uma revisão da literatura científica e atual sobre o assunto.

\section{Metodologia}

Para essa revisão foram consultadas as bases de dados MEDLINE, SciELO, LILACS, ISI e Standford University, utilizando-se a combinação dos seguintes descritores: "obesidade", "complicações", "criança", "dores músculo-esqueléticas", "postura", "alterações ortopédicas", "marcha", "children obesity”, "orthopedic disorders", "posture", "gait" e "musculoskeletal pain".

Foram encontrados 18 artigos que abordavam direta ou indiretamente no corpo do texto os temas: dor ou problemas músculo-esqueléticos e/ou alterações posturais em crianças e adolescentes com sobrepeso e obesidade. Desses 18 artigos, 8 são de revisão e 10 são artigos originais. Foram incluídos somente artigos na língua inglesa e portuguesa, sendo que dois são em português e o resto em inglês. O período de publicação foi de 1994 a 2007. Todos os artigos foram publicados em revistas indexadas. 
Outras duas referências analíticas clássicas (livros) publicadas em 1995 e 2004 foram incluídas na revisão e, além dessas, mais alguns artigos relacionados à definição e prevalência de obesidade infanto-juvenil, para dar substrato à introdução

\section{Resultados e discussão}

A obesidade infantil é considerada um problema de saúde pública, por quase sempre estar relacionada a um grande número de situações patológicas, nas quais se incluem disfunções do aparelho locomotor (5). Essas disfunções não estão presentes somente em obesos, mas sua prevalência é maior graças ao aumento de massa corporal e pelo consequente aumento das necessidades mecânicas regionais (11). A relação entre obesidade e sistema musculoesquelético em crianças não é clara, mas se acredita que a obesidade afeta o aparelho locomotor, tanto de forma estrutural como funcional (12).

O tecido ósseo remodela-se de acordo com a carga exercida sobre ele; portanto, durante a infância os ossos possuem maior quantidade de colágeno e por isso são mais flexíveis, sendo mais tolerantes à deformação plástica e menos resistentes à compressão. Dessa forma, quando há um aumento da sobrecarga, os indivíduos em fase de crescimento são mais susceptíveis às deformações. Sabe-se que em indivíduos com peso normal, a maioria das articulações da extremidade inferior é exposta a uma força de reação de aproximadamente três a seis vezes o peso do corpo durante a locomoção. Em conseqüência disso, os indivíduos obesos experimentam maior sobrecarga em suas articulações que os indivíduos com peso normal (13).

Alterações posturais e dores músculo-esqueléticas são as complicações mais comuns da obesidade (14). Segundo Bruschini e Néri (15), a presença de abdômen protuso nos obesos desloca anteriormente o centro de gravidade, ocasionando adaptações na coluna vertebral e membros inferiores. Na coluna, geralmente há um aumento da lordose lombar com cifose dorsal compensatória, a qual leva à hiperlordose cervical e anteriorização da cabeça. Nos membros inferiores, podem estar presentes a anteversão pélvica associada à rotação interna dos quadris, joelhos valgos e pés planos.

Essa postura, que inicialmente é temporária e flexível, surge de forma compensatória para melhorar a estabilidade e é considerada patológica a partir do momento em que se torna fixa, resultante de adaptações musculares e retrações cápsulo-ligamentares, podendo causar dores no sistema osteomioarticular (10).

Campos et al. (16) sugere que o esforço para manter a estabilidade corporal causado pelo excesso de massa corporal aumenta as necessidades mecânicas do corpo e, consequentemente, aumenta o gasto de energia, desfavorecendo os indivíduos obesos a realizar suas atividades físicas habituais, inclusive a marcha, propiciando a instalação de quadros dolorosos. Outros estudos mostram que os jovens obesos apresentam maior chance de terem dores e problemas ortopédicos, principalmente em membros inferiores e coluna vertebral $(10,17)$.

Pinto et al. (10) examinaram crianças obesas e não obesas, e 55\% das crianças com excesso de peso apresentavam joelhos valgos, comparado com apenas $2 \%$ da população eutrófica. Corroborando com o trabalho de Campos et al. (16), os joelhos hiperestendidos e a hiperlordose lombar também estavam presentes.

A grande incidência de joelho valgo em obesos se explica pelo aumento da anteversão pélvica, que causa rotação interna dos quadris. Isso, associado ao acúmulo de gordura na região das coxas, causa o afastamento da região dos maléolos, promovendo a abertura do compartimento medial e uma hiperpressão no compartimento lateral do joelho. Com o tempo e o desenvolvimento, ocorre um crescimento desigual entre os dois compartimentos, conduzindo à instalação de uma deformidade fixa (15).

Campos et al. (16) afirmam que uma angulação em valgo do joelho pode levar a uma angulação em valgo de retropé e, em decorrência disso, ao desabamento do arco plantar medial, causando o pé pronado ou o pé plano. Pfeiffer et al. (18) analisaram a prevalência de pés planos em pré-escolares e aqueles que estavam com sobrepeso apresentaram $27 \%$ a mais de risco de terem essa condição, enquanto que as crianças obesas tiveram três vezes mais chances de possuírem pés planos em comparação com os eutróficos. 
Taylor et al. (19) compararam o alinhamento de membros inferiores e a prevalência de dores músculoesqueléticas em crianças obesas e não obesas e constataram que os obesos tinham uma prevalência maior de mau alinhamento dos membros inferiores, principalmente em valgo de joelho, e sentiam mais dores articulares, principalmente nos joelhos, seguidos pela coluna lombar. Outros autores utilizaram escalas de dor para avaliar obesos e não obesos e identificaram as dores articulares como queixas frequentes dos adolescentes, sendo mais uma vez as dores nos membros inferiores (44\% dos obesos) e coluna lombar ( $30 \%$ dos obesos) as mais relatadas $(10,20)$.

A relação entre dor músculo-esquelética e obesidade infantil não é bem esclarecida, mas existem algumas hipóteses, como sobrecarga articular e mau alinhamento articular. O joelho valgo pode ser uma causa de dor nos joelhos, pois o aumento da angulação tíbio-femoral favorece o surgimento de síndromes dolorosas da articulação fêmoro-patelar e também o deslocamento lateral da patela. O pé plano, por sua vez, não é biomecanicamente eficiente e pode aumentar a tensão da fáscia plantar, causando fadiga e dores no calcâneo e panturrilhas durante as atividades motoras diárias (13).

Além disso, existe a hipótese de que o aumento na adiposidade seja capaz de alterar fatores hormonais e fatores de crescimento, interferindo no metabolismo da cartilagem articular e osso subcondral (9). Além da cartilagem, a obesidade parece ter efeito sobre outros tecidos moles, como tendão e fáscia (13).

Baseado nisso, Ding et al. (21) avaliaram a relação entre o índice de massa corporal (IMC) e a morfologia da cartilagem articular do joelho em adultos, por meio de exames de imagem, e concluíram que o aumento no IMC favorece o aparecimento de defeitos na cartilagem articular e o espessamento do osso subcondral da tíbia. Essas alterações estruturais da articulação são características de patologias degenerativas do joelho, como as osteoartrites, comuns em jovens mulheres (9). No entanto, Jones et al. (22) não encontraram diferenças significativas no volume de cartilagem articular entre crianças obesas e não obesas.

Campos et al. (16) acreditam que o mau alinhamento dos membros inferiores, associado ao aumento da massa de gordura, proporciona um maior gasto de energia para realizar as atividades motoras diárias. Chen et al. (17) analisaram a eficiência da caminhada e do stepping em adultos, através de calorimetria, e a sua relação com a massa de gordura corporal. Observaram que os obesos foram menos eficientes que os não obesos durante uma caminhada em velocidade normal.

Nantel et al. (23) estudaram as estratégias de locomoção em crianças obesas e não obesas e descobriram que as primeiras diminuem a fase de balanço durante a marcha. McGraw et al. (24) também examinaram a marcha de crianças obesas e não obesas, em diferentes velocidades e confirmaram que o grupo de obesos realizava um aumento do duplo apoio e uma diminuição na fase de balanço da marcha, provavelmente para ajudar a manter o equilíbrio e a estabilidade postural. Esses mesmos autores investigaram a estabilidade postural dessas crianças por meio de estabilometria e os resultados sugerem que o aumento de gordura corporal diminui tanto a estabilidade ântero-posterior quanto a látero-lateral, sendo esta última mais evidente. Isso pode sugerir que índices de massa corporal maiores demandam maiores deslocamentos para manter o equilíbrio $(25,26)$, corroborando com os achados de Deforche et al. (27), que verificaram que os obesos apresentaram diminuição na capacidade de realizar diversas atividades de equilíbrio estático e dinâmico, quando comparados aos com peso normal.

Klenerman (28) e Zonfrillo et al. (29) atentam ainda ao fato de que as crianças podem ser mais facilmente lesionadas durante atividades esportivas do que indivíduos com maturação esquelética completa, pois seus ossos são mais porosos e frágeis, por causa das epífises de crescimento. Dessa forma, Calvete (11) complementa que, no exercício físico desenvolvido para jovens obesos, é importante considerar o peso corporal desses indivíduos como uma sobrecarga durante o esforço físico. Ainda, essas crianças e adolescentes são mais suscetíveis a apresentar persistência de sintomas após uma lesão articular, quando comparadas com seus pares não obesos (30).

Considerando que o corpo responde aos estímulos a ele causados pela obesidade, ele também irá responder aos estímulos que tentem minimizar seus efeitos no sistema músculo-esquelético. Por isso, é importante que a intervenção nos indivíduos obesos com problemas ortopédicos seja realizada na fase de maturação, pois nessa fase melhores resultados poderão ser obtidos em comparação com o período o processo de crescimento já está completo e as deformidades, possivelmente instaladas (16). 


\section{Considerações finais}

Analisando os trabalhos estudados, pode-se observar que os indivíduos obesos são mais predispostos a apresentar problemas ortopédicos do que indivíduos eutróficos. Dentre os principais problemas estão as alterações posturais, nas quais se incluem predominantemente a hiperlordose lombar, os joelhos valgos, os joelhos hiperestendidos e os pés planos. No âmbito articular, destacam-se as dores na coluna lombar e membros inferiores, principalmente nos joelhos.

Não há consenso sobre as causas dessas desordens; no entanto, acredita-se que elas acontecem pela associação de vários fatores, como aumento da sobrecarga articular em conjunto com uma estrutura esquelética ainda em formação, diminuição da estabilidade postural e aumento do gasto energético para realizar as atividades habituais.

Como esses problemas podem acontecer em idade precoce, é imprescindível que essas crianças sejam tratadas com acompanhamento nutricional, fisioterapia e atividade física controlada, para prevenir futuras complicações e melhorar as doenças existentes.

Poucos artigos originais foram encontrados sobre o assunto, o que mostra a necessidade da elaboração de mais artigos originais nessa faixa etária. No entanto, essa revisão torna-se importante para fomentar o conhecimento dos profissionais de saúde e estimular mais pesquisas nessa área.

\section{Referências}

1. Rudolf MCJ. The obese child. Archives of Disease in Childhood. 2004;89:57-62.

2. Cabreira MAS, Filho WJ. Obesidade em idosos: prevalência, distribuição e associação com hábitos e co-morbidades. Arq Bras Endoc Metabol. 2001;45(5):494-501.

3. He Q, Ding ZY, Fong Y, Kalberg J. Blood pressure is associated with body mass index in both normal and obese children. Hipertension. 2000;36:165-70.

4. Reilly JJ. Obesity in childhood and adolescence: evidence based clinical and public health perspectives. Postgrad Med J. 2006;82(969):429-37.

5. Kiess W, Galler A, Reich A, Muller G, Kapellen T, Deutscher R, et al. Clinical aspects of obesity in childhood and adolescence. Obes Rev. 2001;2(1):29-36.

6. Instituto Brasileiro de Geografia e Estatística. Pesquisa de orçamentos familiares 2002-2003. Antropometria e análise do estado nutricional de crianças e adolescentes no Brasil. Rio de janeiro: IBGE; 2006.

7. Leite N, Milano GE, Cieslak F, Lopes WA, Rodacki A, Radominski, RB. Efeito do exercício físico e da orientação nutricional na síndrome metabólica em adolescentes obesos. Rev Bras Fisioter. 2009;13(1):73-81.

8. DietzWH. Health consequences of obesity in youth: childhood predictors of adult disease. Pediatrics. 1998;101(3 Pt2):518-25.

9. Powel A, Teichtahl AJ, Wluka AE, Cicuttini FM. Obesity: a preventable risk factor for large joint osteoarthritis wich may act through biomechanical factors. Br J Sports Med. 2005;39(1):4-5.

10. Pinto ALS, Holanda PMB, Radu AS, Villares MF, Lima FR. Musculoskeletal findings in obese children. J Paediatr Child Health. 2006;42:341-4.

11. Calvete SA. A relação entre alteração postural e lesões esportivas em crianças e adolescentes obesos. Motriz. 2004;10(2):67-72.

12. Chan G, Chen CT. Musculoskeletal effects of obesity. Curr Opin Pediatr. 2009;21(1):65-70.

13. Wearing SC, Henning EM, Byrne NM, Steele JR, Hills AP. Musculoskeletal disorders associated with obesity: a biomechanical perspective. Obes Rev. 2006;7(3):239-50. 
14. Dâmaso AR, Teixeira LR, Curi CMON. Atividades motoras na obesidade. In: Fisberg M, editor. Atualização em obesidade na infância e adolescência. São Paulo: Atheneu; 2004. p. 91-9.

15. Bruschini S. Nery CAS. Aspectos ortopédicos da obesidade na infância e adolescência. In: Fisberg M. Obesidade na infância e adolescência. São Paulo: Fundação BYK; 1995. p. 105-25.

16. Campos FS, Silva AS, Anhesim GA. Alterações posturais e abordagem fisioterapêutica em crianças e adolescentes obesos. In: Fisberg M. Atualização em obesidade na infância e adolescência. São Paulo: Atheneu; 2004. p. 131-41.

17. Chen KY, Acra SA, Donahue CL, Sun M, Buchowski MS. Efficiency of walking and stepping: relationship to body fatness. Obes Res. 2004;12(6):982-9.

18. Pfeiffer M, Kotz R, Ledl T, Hauser G, Sluga M. Prevalence of flat foot in pre-school children. Pediatrics. 2006;118(2):634 -9.

19. Taylor ED, Theim KR, Mirch MC, Ghorbani S, Tanofsky-Kraffy M, Adler-Wailes T, et al. Orthopedics complications of overweight in children and adolescents. Pediatrics. 2006;117(6):2167-74.

20. Stovitz SD, Pardee PE, Vazquez G, Duval S, Schwimmer JB. Musculoskeletal pain in obese children and adolescents. Acta Pædiatrica. 2008;97(4):489-93.

21. Ding C, Cicutini F, Scott F, Cooley H, Jones, G. Knee structural alteration and BMI: a cross-sectional study. Obes Res. 2005;13(2):350-61.

22. Jones G, Ding CH, Hynes KL, Ma D, Glisson M, Cicuttini FM. Knee articular cartilage development in children: a longitudinal study of the effect of gender, growth, Tanner stage and physical activity. Pediatr Res. 2003;54:230-6.

23. Nantel J, Brochu M, Prince F. Locomotor strategies in obese and non-obese children. Obesity. 2006;14(10):1789-94.

24. Mcgraw B, Mcclenaghan BA, Williams HG, Dickerson J, Ward DS. Gait and postural stability in obese and nonobese prepubertal boys. Arch Phys Med Rehabil. 2000;81(4):484-9.

25. Greeve J, Alonso A, Bordini ACPG, Camanho GL. Correlations between body mass index and postural balance. Clinics. 2007;62(6):717-20.

26. Hills AP, Parker, AW. Gait characteristics of obese children. Arch Phys Med Rehabil. 1991;72(6):403-7.

27. Deforche BI, Hills AP, Worringham CJ, Davies, PSW, Murphy AJ, Bouckaert JJ, et al. Balance and postural skills in normal-weight and overweight prepubertal boys. Int J Pediatr Obes. 2009;4(3):175-82.

28. Klenerman L. ABC of sports medicine: musculoskeletal injuries in child athletes. BMJ. 1994;308(6943):1556-9.

29. Zonfrillo MR, Seiden JA, House EM, Shapiro ED, Dubrow R, Baker MD, et al. The association of overweight and ankle injuries in children. Ambul Pediatr. 2008;8(1):66-9.

30. Timm NL, Grupp-Phelan J, Ho ML. Chronic ankle morbidity in obese children following an acute ankle injury. Arch Pediatr Adolesc Med. 2005;159(1):33-6.

Recebido: 30/07/2008

Received: 07/30/2008

Aprovado: 06/10/2009

Approved: 10/06/2009

Revisado: 12/05/2010

Reviewed: 05/12/2010 